PREPARED FOR THE U.S. DEPARTMENT OF ENERGY, UNDER CONTRACT DE-AC02-76CH03073

PPPL-3739 Rev

PPPL-3739 Rev

UC-70

Localized Measurement of Turbulent Fluctuations in Tokamaks with Coherent Scattering of Electromagnetic Waves

by

E. Mazzucato

August 2002

Revised: December 2002

$N_{\substack{\text { PRInCETOn PLASMA } \\ \text { PHYSIES LABORATORY }}}^{D}$

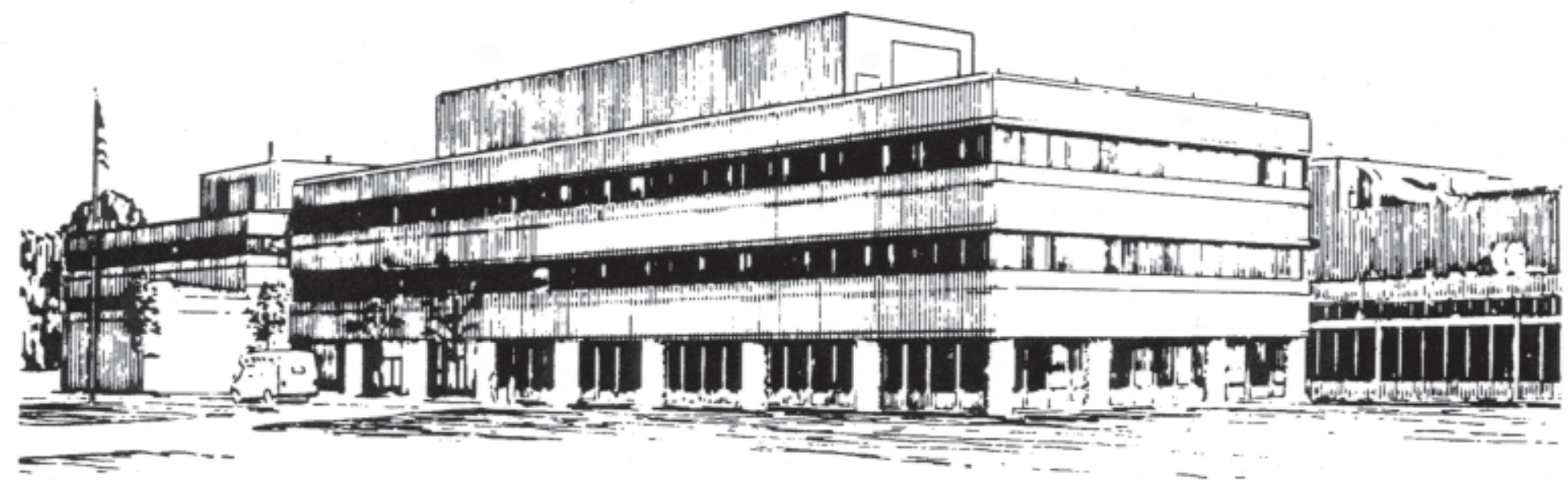

PRINCETON PLASMA PHYSICS LABORATORY PRINCETON UNIVERSITY, PRINCETON, NEW JERSEY 


\section{PPPL Reports Disclaimer}

This report was prepared as an account of work sponsored by an agency of the United States Government. Neither the United States Government nor any agency thereof, nor any of their employees, makes any warranty, express or implied, or assumes any legal liability or responsibility for the accuracy, completeness, or usefulness of any information, apparatus, product, or process disclosed, or represents that its use would not infringe privately owned rights. Reference herein to any specific commercial product, process, or service by trade name, trademark, manufacturer, or otherwise, does not necessarily constitute or imply its endorsement, recommendation, or favoring by the United States Government or any agency thereof. The views and opinions of authors expressed herein do not necessarily state or reflect those of the United States Government or any agency thereof.

\section{Availability}

This report is posted on the U.S. Department of Energy's Princeton Plasma Physics Laboratory Publications and Reports web site in Fiscal Year 2003. The home page for PPPL Reports and Publications is: http://www.pppl.gov/pub_report/

DOE and DOE Contractors can obtain copies of this report from:

U.S. Department of Energy

Office of Scientific and Technical Information

DOE Technical Information Services (DTIS)

P.O. Box 62

Oak Ridge, TN 37831

Telephone: (865) 576-8401

Fax: (865) 576-5728

Email: reports@adonis.osti.gov

This report is available to the general public from:

National Technical Information Service

U.S. Department of Commerce

5285 Port Royal Road

Springfield, VA 22161

Telephone: $1-800-553-6847$ or

(703) $605-6000$

Fax: (703) 321-8547

Internet: http://www.ntis.gov/ordering.htm 


\title{
Localized measurement of turbulent fluctuations in tokamaks with coherent scattering of electromagnetic waves
}

E. Mazzucato ${ }^{1}$

Princeton Plasma Physics Laboratory, Princeton, New Jersey 08543, USA

\begin{abstract}
Localized measurements of short-scale turbulent fluctuations in tokamaks are still an outstanding problem. In this paper, the method of coherent scattering of electromagnetic waves for the detection of density fluctuations is revisited. Results indicate that the proper choice of frequency, size and launching of the probing wave can transform this method into an excellent technique for high-resolution measurements of those fluctuations that plasma theory indicates as the potential cause of anomalous transport in tokamaks. The best spatial resolution can be achieved when the range of scattering angles corresponding to the spectrum of fluctuations under investigation is small. This favors the use of high frequency probing waves, such as those of far infrared lasers. The application to existing large tokamaks is discussed.
\end{abstract}

\footnotetext{
${ }^{1}$ Electronic mail: mazzucato@pppl.gov
} 


\section{INTRODUCTION}

Understanding the mechanism of anomalous transport in tokamaks is one of the great challenges of fusion research. Indeed, since many explanations of this phenomenon are based on some type of turbulence, ${ }^{1,2}$ understanding anomalous transport is tantamount to understanding plasma turbulence.

The main difficulty in drawing any firm conclusion from fluctuation measurements is their scarcity and limitations. For example, wave scattering measurements, that were so prominent in early fluctuation studies, ${ }^{3-7}$ have a poor spatial resolution - very often larger than the plasma minor radius. The method of Beam Emission Spectroscopy ${ }^{8}$ requires a perturbing neutral beam, has serious difficulties in detecting plasma fluctuations in the central region of large tokamaks, and is sensitive only to relatively large-scale fluctuations. The interpretation of microwave reflectometry is extremely difficult, and it cannot be done unambiguously. ${ }^{9,10}$ The inevitable conclusion is that we must improve the capability of diagnostic tools for advancing our understanding of plasma turbulence. Ideally, what is needed is a method capable of detecting all types of short-scale fluctuations. Indeed this is a daunting task given the variety of fluctuations in tokamak plasmas - from the Ion Temperature Gradient (ITG) mode and the Trapped Electron Mode (TEM) with the scale of the ion Larmor radius, to the Electron Temperature Gradient (ETG) mode with the scale of the Larmor radius of electrons. As an example, for the typical plasma conditions of the Tokamak Fusion Test Reactor, ${ }^{8,9}$ the wave number of possible fluctuations could vary from $1-2 \mathrm{~cm}^{-1}$ for the ITG mode, to $\sim 50 \mathrm{~cm}^{-1}$ for the ETG mode.

In this paper, we will revisit the method of coherent scattering of electromagnetic waves for the detection of plasma density fluctuations. After a brief review of the theory of wave scattering, we will discuss how to optimize this technique for performing highresolution measurements of those fluctuations that plasma theory indicates as the potential cause of anomalous transport in tokamaks. 


\section{COHERENT SCATTERING OF EM WAVES}

Coherent scattering of electromagnetic waves is a powerful technique, capable of providing a direct measure of the spectral power density of turbulent fluctuations. It was employed extensively in early studies of plasma turbulence, including the first detection of short-scale density fluctuations in tokamaks. 3,4

The scattering process is characterized by the differential cross section $\sigma=\sigma_{o} S(\boldsymbol{k}, \omega)$, where $\sigma_{o}=\left(e^{2} / m c^{2}\right)^{2}$ is the Thomson cross section and $S(\boldsymbol{k}, \omega)$ is the spectral density of plasma density fluctuations. The frequency $\omega$ and the wave vector $\boldsymbol{k}$ must satisfy the energy and momentum conservation, i.e., $\omega=\omega^{s}-\omega^{i}$ and $\boldsymbol{k}=\boldsymbol{k}^{s}-\boldsymbol{k}^{i}$, where the superscripts $s$ and $i$ refer to the scattered and the incident wave, respectively. Since for the topic of this note $\omega^{s} \approx \omega^{i}$ and $k^{s} \approx k^{i}$, the scattering angle $\theta$ must satisfy the Bragg equation

$$
k=2 k^{i} \sin (\theta / 2) .
$$

The instrumental resolution of scattering measurements is limited by the size of the probing and scattered beams, that in this paper we will assume having a Gaussian amplitude profile $A\left(r_{\perp}\right)=\exp \left(-r_{\perp}^{2} / w^{2}\right)$, where $r_{\perp}$ is a radial coordinate perpendicular to the direction of propagation and $w$ the beam radius. The wave number resolution of measured fluctuations depends on the beam spectrum $G\left(\kappa_{\perp}\right)=\exp \left(-\kappa_{\perp}^{2} / \Delta^{2}\right)$, where $\Delta=2 / w$ and $\kappa_{\perp}$ is the wave number perpendicularly to the direction of propagation. For example, we get $\Delta=0.5 \mathrm{~cm}^{-1}$ for $w=4 \mathrm{~cm}$, which is an adequate resolution when compared to the wave number of expected fluctuations. However, if we take the linear dimension of the common region between the probing and the scattered beam as a measure of the spatial resolution, we conclude that it is very difficult to perform spatially resolved fluctuation measurements in tokamaks using coherent scattering of electromagnetic waves. As an example, for a probing wavelength of $\sim 1 \mathrm{~mm}$ and a beam radius of $4 \mathrm{~cm}$, the spatial resolution $\delta r \approx 2 k^{i} w / k=50 \mathrm{~cm}$ for $k=10 \mathrm{~cm}^{-1}$, which is clearly unsatisfactory since most of the turbulent activity in large tokamaks occurs at much lower wave 
numbers. However, such an estimate of spatial resolution does not take into account the spatial variation of magnetic field lines. Indeed, since for the short-scale density fluctuations of tokamaks $\boldsymbol{k} \cdot \boldsymbol{B}<<k B$, i.e.,

$$
\boldsymbol{k} \cdot \boldsymbol{B} \approx 0,
$$

a change in direction of the magnetic field can modify the instrumental selectivity function by detuning the scattering receiver. This can be easily understood when the probing wave propagates perpendicularly to the magnetic surfaces and the scattering angle is small (Fig. 1). From the Gaussian spectrum $G\left(\kappa_{\perp}\right)$ of the previous paragraph one can readily obtain the instrumental selectivity function of the receiving antenna ${ }^{7}$

$$
F(\boldsymbol{r})=\exp \left[-(2 k \sin (\xi(r) / 2) / \Delta)^{2}\right],
$$

where $\xi(r)$ is the change in pitch angle of magnetic field lines starting from the beam location where scattered waves are detected with the maximum efficiency, i.e., where the receiving antenna is pointing to.

From Eq. (3), we get a spatial resolution of $\delta r \approx 2 \Delta / k\langle d \xi / d r\rangle$ in the radial plasma direction (where $\langle d \xi / d r\rangle$ is the average radial derivative of the magnetic pitch angle inside the scattering region). Compared to the previous estimate of spatial resolution, Eq. (3) does not depend on the wave number of the probing wave. This is very advantageous for scattering of far infrared red waves, since for these Eq. (3) gives an instrumental resolution which is substantially better than the linear dimensions of the common region between the probing and scattered beams. Unfortunately, for typical tokamak plasmas where $d \xi / d r<0.2 \mathrm{rad} / \mathrm{m}$, such an improvement in spatial resolution is not always sufficient for our goals. This is demonstrated in Fig. 2, which shows the instrumental selectivity functions for the case of a Joint European Torus (JET) discharge (minor/major radius $=0.95 / 2.85 \mathrm{~m}$, toroidal magnetic field $=3.4 \mathrm{~T}$, plasma current $=4 \mathrm{MA}$ ) where a Gaussian probing beam with a radius of $4 \mathrm{~cm}$ is launched perpendicularly to the magnetic surfaces from a point on the high field side of the equatorial plane. Even though such a scattering arrangement - indeed very impractical and difficult to implement - maximizes 
the benefits of magnetic shear, the instrumental selectivity functions of Fig. 2 are very broad, and would therefore result in poorly localized scattering measurements.

In this paper, we will consider the general case of a probing beam propagating at an arbitrary angle to the magnetic field. The idea behind this is that, when the probing beam forms a small angle with the toroidal magnetic field, the conditions for coherent wave scattering [Eqs. (1) and (2)] become strongly dependent on the toroidal angle. In addition, a probing beam nearly tangent to the magnetic surfaces has the additional advantage of minimizing the extent of the scattering region in the radial plasma direction.

\section{OBLIQUE PROPAGATION}

To derive the general expression of the instrumental selectivity function for an arbitrary propagation of the probing beam, we use a system of orthogonal coordinates $(u, v, t)$ having the $u$-axis parallel to the equatorial plane and the $t$-axis parallel to the wave vector $\boldsymbol{k}^{i}$, and we define the angle $\varphi$ with

$$
k_{u}^{s}=k^{i} \sin \theta \cos \varphi, \quad k_{v}^{s}=k^{i} \sin \theta \sin \varphi, k_{t}^{s}=k^{i} \cos \theta .
$$

Let us now consider scattered waves from two regions of the probing beam with identical scattering angles but different wave vectors $\boldsymbol{k}_{1}^{s}$ and $\boldsymbol{k}_{2}^{s}$ (Fig. 3). From Eq. (4), we get

$$
\frac{\boldsymbol{k}_{1}^{s} \cdot \boldsymbol{k}_{2}^{s}}{k^{i 2}}=1-2 \sin ^{2}(\delta \varphi / 2) \sin ^{2} \theta
$$

where $\delta \varphi=\varphi_{2}-\varphi_{1}$. Suppose, then, that the detection system is tuned for the measurement of scattered waves from the first region. Those from the second region will be collected with a relative efficiency

$$
F=\exp \left[-(2 k \sin (\delta \varphi / 2) / \Delta)^{2}\right],
$$

where we have used the Bragg condition with $\theta^{2}<<1$. For propagation perpendicularly to the magnetic surfaces, $\varphi$ becomes the magnetic pitch angle (apart from an additive constant), and Eq. (6) coincides with Eq. (3). 
Finally, the angle $\varphi$ is obtained from Eq. (2), which gives

$$
\cos \varphi=\frac{B_{u} B_{t}(1-\cos \theta) \pm\left[B_{u}^{2} B_{t}^{2}(1-\cos \theta)^{2}-B_{\perp}^{2}\left(B_{t}^{2}(1-\cos \theta)^{2}-B_{v}^{2} \sin ^{2} \theta\right)\right]^{1 / 2}}{B_{\perp}^{2} \sin \theta}
$$

and

$$
\sin \varphi=\frac{B_{t}(1-\cos \theta)-B_{u} \sin \theta \cos \varphi}{B_{v} \sin \theta}
$$

with $B_{\perp}^{2}=B_{u}^{2}+B_{v}^{2}$.

In the next section, we will use these equations for showing how to perform highresolution measurements of turbulent fluctuations in tokamaks using coherent scattering of electromagnetic waves.

\section{HIGH RESOLUTION FLUCTUATION MEASUREMENTS}

We begin with the scattering configuration of Fig. 4, where short-scale fluctuations in a tokamak plasma similar to that of Fig. 2 are probed with a Gaussian beam having a frequency of $3 \times 10^{11} \mathrm{~Hz}$. The initial direction of the probing beam is parallel to the $(x z)$ plane and makes a small angle $(\gamma)$ of $-4.5^{\circ}$ with the equatorial plane (with the negative sign indicating a downward direction). Finally, the launching point is chosen so that the beam trajectory in vacuum crosses the equatorial plane at $x=0$, which is also where the beam has a minimum waist of $w_{0}=4 \mathrm{~cm}$. The beam ray trajectories, which are displayed in Fig. 4 on both the equatorial ( $x y)$ and the poloidal $(z r)$ plane, are from a ray tracing code 11 including both plasma refraction and first order diffraction effects. However, at first we will consider a low density plasma and neglect the bending of the beam due to wave refraction. Furthermore, since $\left(2 x / k^{i} w_{0}^{2}\right)^{2} \ll<1$, diffraction is also negligible and consequently the beam radius remains nearly constant $\left(w \approx w_{0}\right)$.

The conditions for coherent wave scattering vary along the path of the probing beam, as illustrated in Fig. 5 which displays the $(u, v)$-components of $\boldsymbol{k}^{s}$ satisfying Eqs. (1) and (2). At each beam location, these components describe an ellipse whose size is a 
decreasing function of the angle $\alpha$ between the wave vector $\boldsymbol{k}^{i}$ and the magnetic field $\boldsymbol{B}$ (Fig. 6). As shown in Fig. 5, the scattering angle depends only on the beam location and the value of $\varphi$. However, not every value of $\theta$ is possible since Eq. (7) imposes the condition

$$
\cos \theta \geq \frac{B_{\|}^{2}-B_{\perp}^{2}}{B_{\|}^{2}+B_{\perp}^{2}},
$$

where $B_{\|}=B_{t}$. When this is satisfied, Eq. (5) gives two values of $\varphi$ for each scattering angle, as shown in Fig. 7 for the scattering geometry of Fig. 4. Once the angle $\varphi$ is known as a function of position and scattering angle, the instrumental selectivity function can be obtained from Eqs. (6) and (8). By assuming that the receiving system is aligned for the detection of scattered waves propagating parallel to the equatorial plane [i.e., using $\varphi_{1}=0^{\circ}$ or $\varphi_{1}=180^{\circ}$ in Eq. (8)], we obtain the instrumental functions of Fig. 8 for fluctuation wave numbers corresponding to the scattering angles of Fig. 7. In Fig. 8, all scattering parameters are calculated for the ray with maximum intensity, i.e., for the central ray. The total instrumental function, then, could be obtained by repeating the calculation for different rays and performing the proper average. However, because of the narrow and parallel beams considered in this paper, the calculated instrumental function does not depend significantly on the chosen ray so that Fig. 8 gives a good representation of the average instrumental function as well.

All instrumental functions in Fig. 8 peak near the point where $\alpha$ has its minimum value $\left(\alpha_{\min }\right)$ and have a width that is a decreasing function of $\alpha_{m i n}$. This is illustrated in Fig. 9, which shows the instrumental function for the conditions of case a) in Fig. 8 but for the specular beam with respect to the equatorial plane, i.e., for the opposite value of $\gamma\left(=4.5^{\circ}\right)$. This demonstrates how a small increase in $\alpha_{\min }$ (Fig. 6) can cause a large broadening of the instrumental function. A reversal of the tokamak plasma current in Fig. 4 would have produced the same result. 
Because of such a strong sensitivity to $\alpha_{\text {min }}$, any refractive bending of the probing beam could also modify the instrumental function. For an evaluation of this phenomenon, we have repeated the previous calculations for a peak density of $5 \times 10^{19} \mathrm{~m}^{-3}$ and the ordinary mode of wave propagation (Fig. 10) for which refraction is not completely negligible. However, since $w$ is much smaller than the plasma density scale length, all rays suffer the same deflection and consequently remain nearly parallel to each other. This causes a shift in the position of the instrumental functions, but no appreciable change in their width (Fig. 11).

The spatial variation of the instrumental function along the beam trajectory may affect the extent of the scattering region in the plasma radial direction and deteriorate the radial localization of scattering measurements. Fortunately, in both Figs. 8 and 11 this is a small effect, and consequently the length of the scattering volume along the plasma radial direction depends only on the radius of the probing beam (i.e., $\delta r \approx \pm w$ ). However, as scattering angles increase (from $1.8^{\circ}$ to $9^{\circ}$ in Fig. 8), the peak of the instrumental function moves away from the midpoint of the probing beam $(x=0)$, causing a spread in the localization of coherent scattering and consequently a deterioration in the spatial resolution of fluctuation measurements. Furthermore, a radial shift of the beam caused by plasma refraction could also cause a radial broadening of the scattering volume. Fortunately, both of these effects are minimized by the use large probing frequencies (i.e., small scattering angles), as demonstrated by Fig. 12 where the case of Fig. 11 is redisplayed for the much larger frequency of $3 \times 10^{13} \mathrm{~Hz}\left(\mathrm{CO}_{2}\right.$ laser $)$. As a result, all instrumental functions peak near the point of tangency to the magnetic surface $(x=0)$.

Ironically, we have reached the point where the length of the scattering volume along the probing beam is too small. Indeed, the narrow instrumental function of case $c$ ) in Fig. 12 causes a decrease in scattered power without contributing to a reduction in the radial extent of the scattering region. A better option, then, is to employ a narrower beam, as in Fig. 13 where a beam with $w=1 \mathrm{~cm}$ is used for the detection of fluctuations with a wave 
number of $10 \mathrm{~cm}^{-1}$. This causes a broadening of the instrumental function and a corresponding increase in scattered power, but no appreciable effect on the instrumental radial resolution which remains equal to the beam diameter and therefore is substantially better than in Fig. 12.

For large fluctuation wave numbers, such as those driven by the ETG mode in tokamaks, the instrumental function could become too narrow even when using small beam radii. This can be cured by decreasing the obliqueness of the probing beam, as illustrated in Fig. 14 where a beam with a radius of $1 \mathrm{~cm}$ and a launching angle of $0^{\circ}$ (instead of $-4.5^{\circ}$ of Fig. 12) is used for the detection of fluctuations with $k=30 \mathrm{~cm}^{-1}$. This causes the value of $\alpha_{\min }$ to increase from $4^{\circ}$ to $9^{\circ}$, producing a broadening of the instrumental function along the beam path, but again without any appreciable effect on the radial dimensions of the scattering volume.

So far we have considered only the measurement of fluctuations at a fixed radial location. Indeed, we could get the radial profile of fluctuations with the scattering arrangement of Fig. 4 by varying the $y$-coordinate of the launching point, or equivalently by rotating the probing beam with respect to the $(x z)$-plane. However, for maintaining constant the instrumental function during the radial scan (i.e., for keeping constant the value of $\alpha_{\text {min }}$ ), we must vary $\gamma$ as well. This is illustrated in Fig. 15, which shows the instrumental selectivity functions at two radial positions $(r=3.1$ and $3.6 \mathrm{~m})$ for fluctuations with $k=5 \mathrm{~cm}^{-1}$ and $\varphi_{1}=0^{\circ}$. The close similarity of the two instrumental functions is achieved using $\gamma=0^{\circ}$ and $\gamma=-12^{\circ}$, respectively.

\section{CONCLUSION}

The results of this paper illustrate how a judicious choice of frequency, size and launching direction of the probing beam could transform coherent scattering of electromagnetic waves into an excellent technique for high-resolution measurements of short-scale turbulent fluctuations in tokamaks. The crucial feature of the proposed 
method is the oblique propagation of the probing beam with respect to the magnetic field. The scattering measurements are localized near the point where the angle between $\boldsymbol{k}^{i}$ and $\boldsymbol{B}$ has a minimum, i.e., where the spatial variation of wave vectors satisfying Eqs. (1) and (2) is more pronounced. This phenomenon does not rely upon the strength of the magnetic shear, but rather on changes in the direction of the toroidal magnetic field inside the scattering region. Furthermore, the best spatial localization is achieved when the range of scattering angles corresponding to the spectrum of fluctuations under investigation is small. This favors the use of high frequency probing waves, such as those of far infrared lasers, which also improves the robustness of this technique by minimizing spurious refractive effects.

Finally, it is important to note that in most of the scattering configurations described in this paper for a large tokamak plasma (Figs. 11-15) the length of the scattering region along the direction of the probing beam is similar to that of previous fluctuation measurements in small tokamaks. ${ }^{3-7}$ Consequently, since for a given field of turbulent fluctuations and probing beam the scattered power is mainly limited by this geometrical parameter, we conclude that the proposed scheme is capable of detecting at least the same level of fluctuations as in previous measurements - but with a much improved radial localization.

In conclusion, the method described in this paper offers a unique opportunity for the experimental study of those fluctuations that plasma theory indicates as the potential cause of anomalous transport in tokamaks.

\section{ACKNOWLEDGEMENT}

The author wishes to thank T. Munsat and H. Park for useful discussions. This work was supported by U.S. DOE Contract No. DE-AC02-76-CHO-3073. 


\section{REFERENCES}

${ }^{1}$ W. Horton, Rev. Mod. Phys. 71, 735 (1999).

2J. W. Connor and H. R. Wilson, Plasma Phys. Control. Fusion 36, 719 (1994).

${ }^{3}$ E. Mazzucato, Phys. Rev. Lett.. 36, 792 (1976).

${ }^{4}$ C. M. Surko and R. E. Slusher, Phys. Rev. Lett.. 37, 1747 (1976).

${ }^{5}$ D. L. Brower, W. A. Peebles, and N. C. Luhmann, Jr., Nucl. Fusion 27, 2055 (1987).

${ }^{6}$ R. Philipona, E. J. Doyle, N. C. Luhmann, Jr., W. A. Peebles, C. Rettig, K. H. Burrell, R.

J. Groebner, H. Matsumoto, and the DIII-D Group, Rev. Sci. Instrum. 61, 3007 (1990).

${ }^{7}$ P. Devynck, X. Garbet, C. Laviron, J. Payan, S. K. Saha, F. Gervais, P. Hennequin, A. Quéméneur, and A. Truc, Plasma Phys. Control. Fusion 35, 63 (1993).

${ }^{8}$ R. Fonck, G. Cosby, R. D. Durst, S. F. Paul, N. Bretz, S. Scott, E. Synakowski, and G. Taylor, Phys. Rev. Lett.. 70, 3736 (1993).

${ }^{9}$ E. Mazzucato and R. Nazikian, Phys. Rev. Lett. 71, 1840 (1993).

${ }^{10}$ E. Mazzucato, Rev. Sci. Instrum. 69, 2201 (1998).

${ }^{11}$ E. Mazzucato, Phys. Fluids B1, 1855 (1989). 


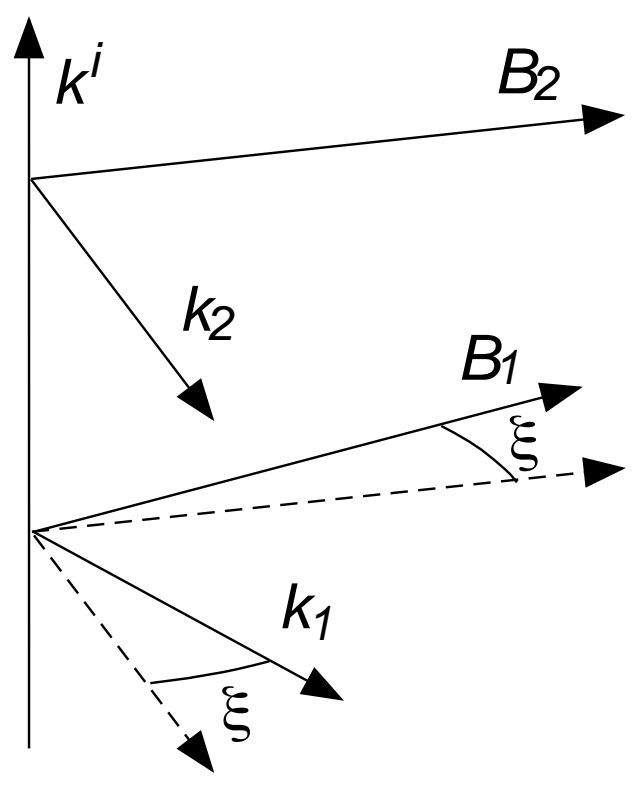

FIG. 1. Magnetic field $\left(\boldsymbol{B}_{1}\right.$ and $\left.\boldsymbol{B}_{2}\right)$ and wave vector $\left(\boldsymbol{k}_{1}\right.$ and $\left.\boldsymbol{k}_{2}\right)$ of detected fluctuations at two locations of a probing beam propagating perpendicularly to the magnetic surfaces. Scattering angles are equal (i.e., $k_{1}=k_{2}$ ) and small (i.e., $\boldsymbol{k}_{1}$ and $\boldsymbol{k}_{2}$ are nearly perpendicular to $\boldsymbol{k}^{i}$ ). 

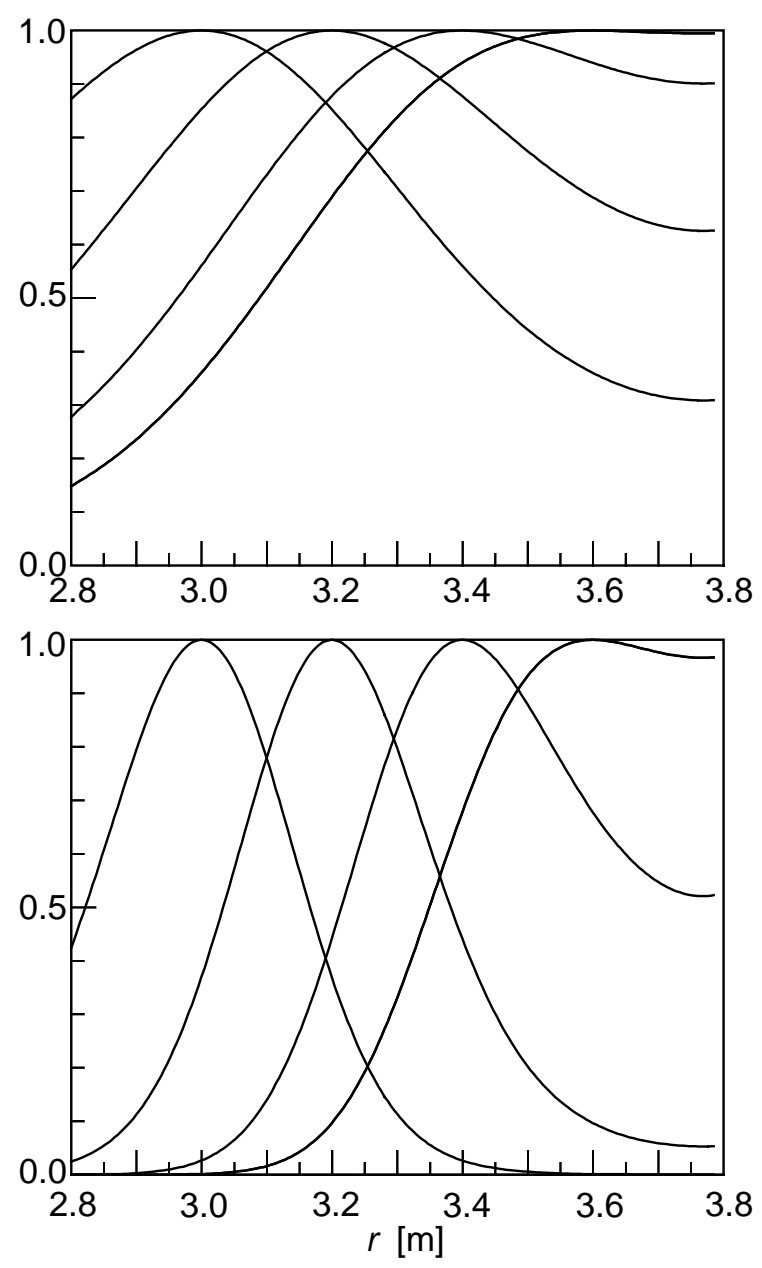

FIG. 2. Instrumental selectivity function for the detection of fluctuations with $k=2$ $\mathrm{cm}^{-1}$ (top) and $k=5 \mathrm{~cm}^{-1}$ (bottom) in a JET-like plasma with a minor radius of 0.95 $\mathrm{m}$. The probing beam has a radius of $4 \mathrm{~cm}$ and propagates on the equatorial plane perpendicularly to the magnetic surfaces. 


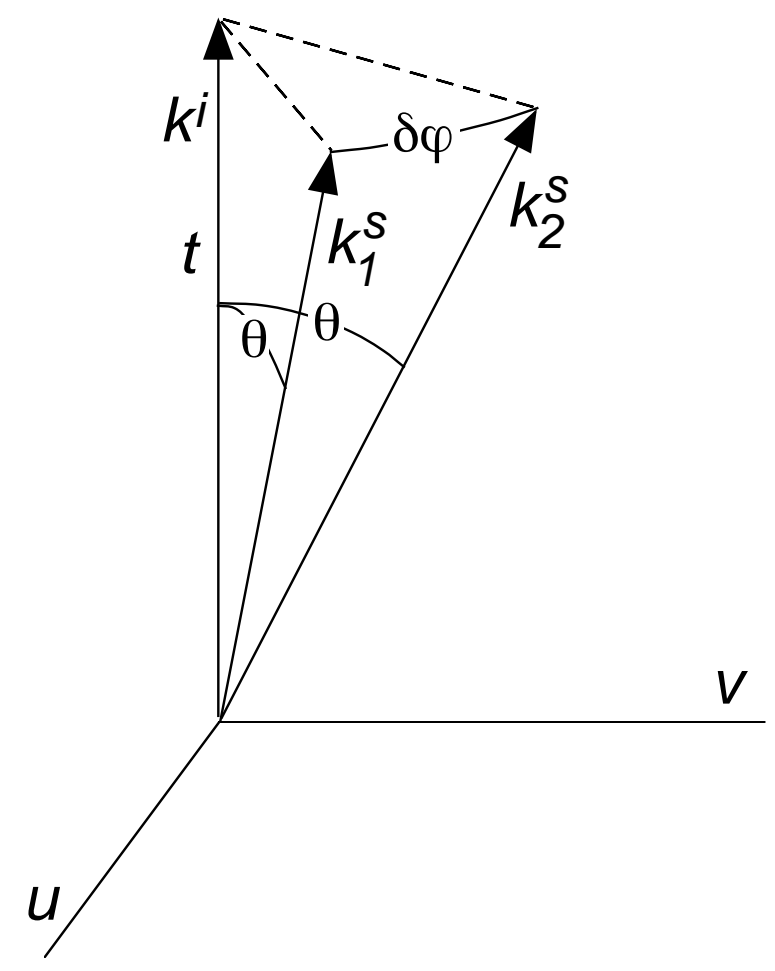

FIG. 3. System of orthogonal coordinates $(u, v, t)$ used for deriving the scattering conditions. The $u$-axis is parallel to the tokamak equatorial plane and the $t$-axis is parallel to $\boldsymbol{k}^{i}$. 

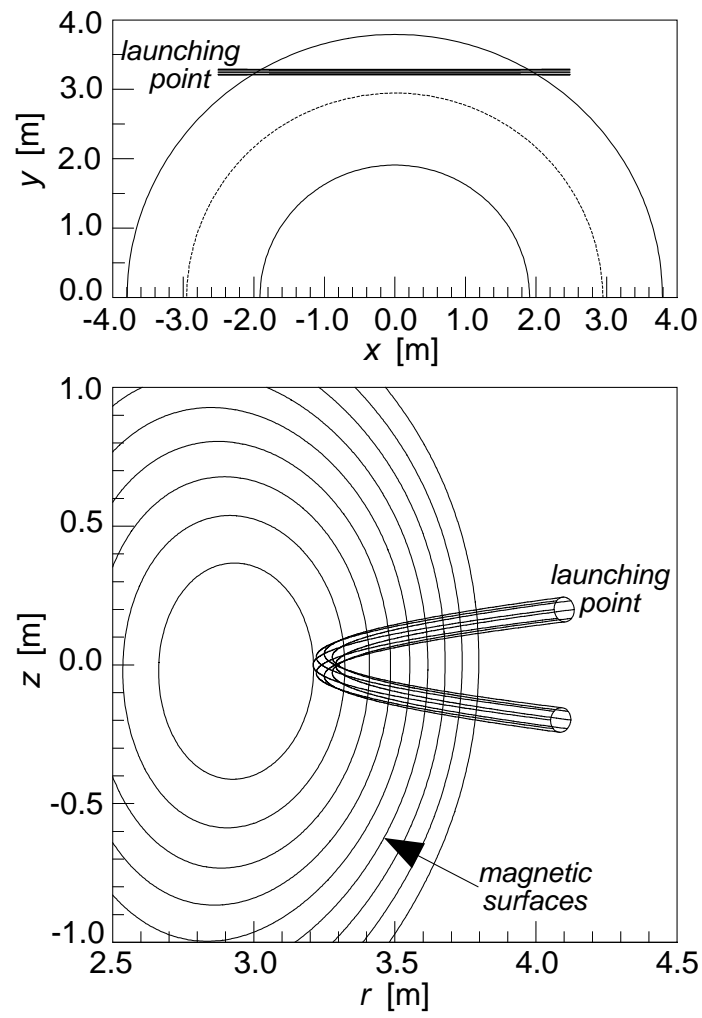

FIG. 4. Toroidal (top) and poloidal (bottom with $r=\left[x^{2}+y^{2}\right]^{1 / 2}$ ) projections of the $1 / e$ amplitude rays of a Gaussian beam propagating in a low density plasma (i.e., negligible refractive effects) with a frequency of $3 \times 10^{11} \mathrm{~Hz}$, radius $4 \mathrm{~cm}$ and launching angle $-4.5^{\circ}$. 


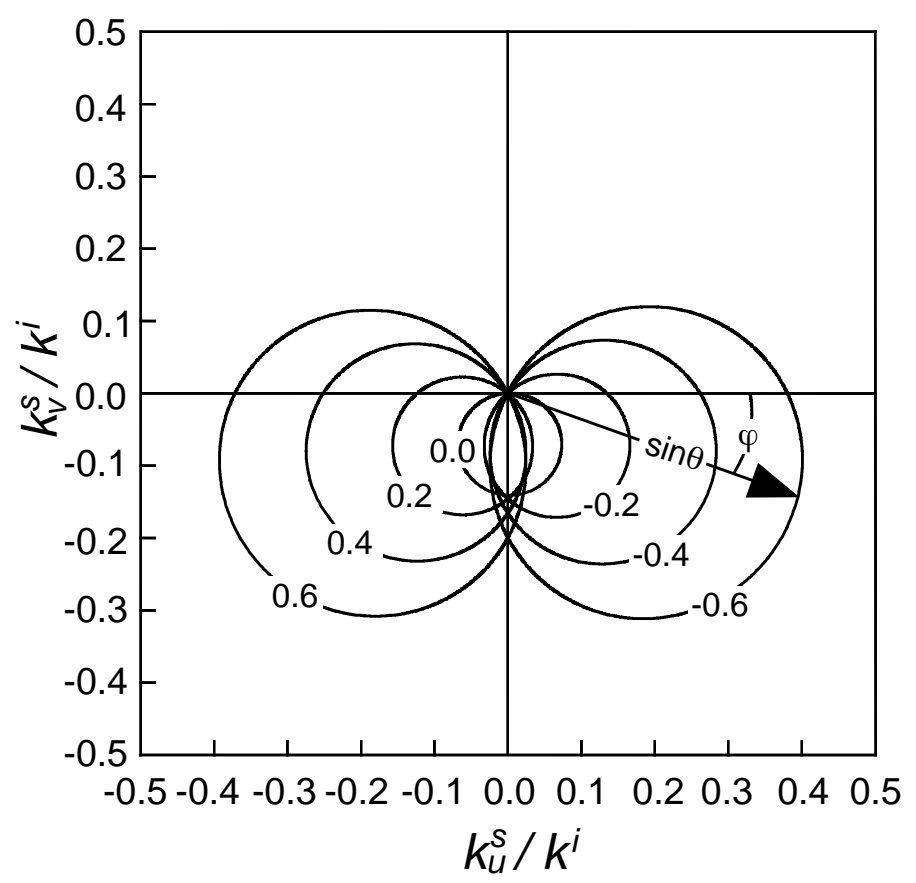

FIG. 5. Normalized (u,v)-components of $\boldsymbol{k}^{s}$ satisfying Eqs. (1) and (2). The $u$-axis is in the $y$-direction (Fig. 4); parameters are the values of $x$ along the probing beam. 


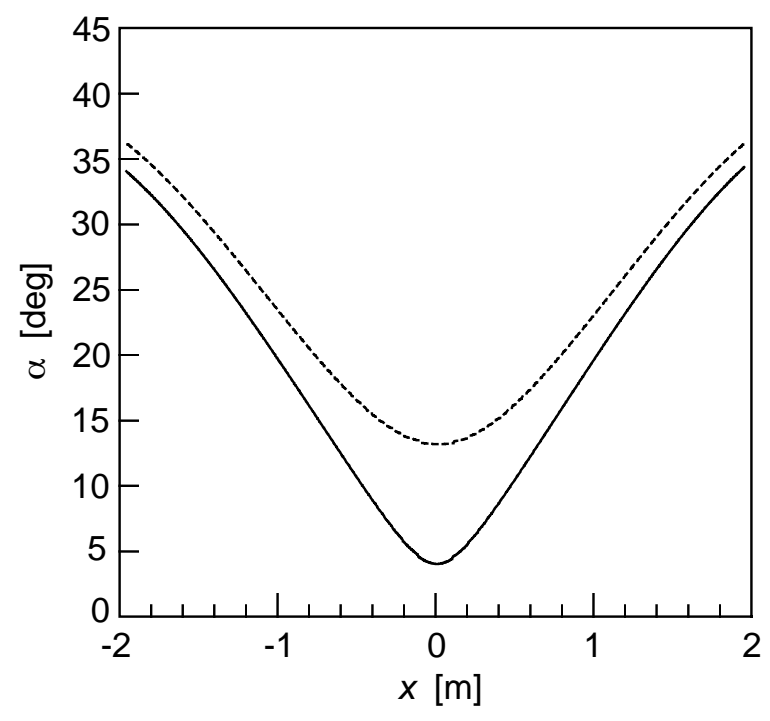

FIG. 6. Angle of probing beam with the magnetic field as a function of position; $\gamma=-4.5^{\circ}$ (solid line) and $\gamma=4.5^{\circ}$ (dashed line). 


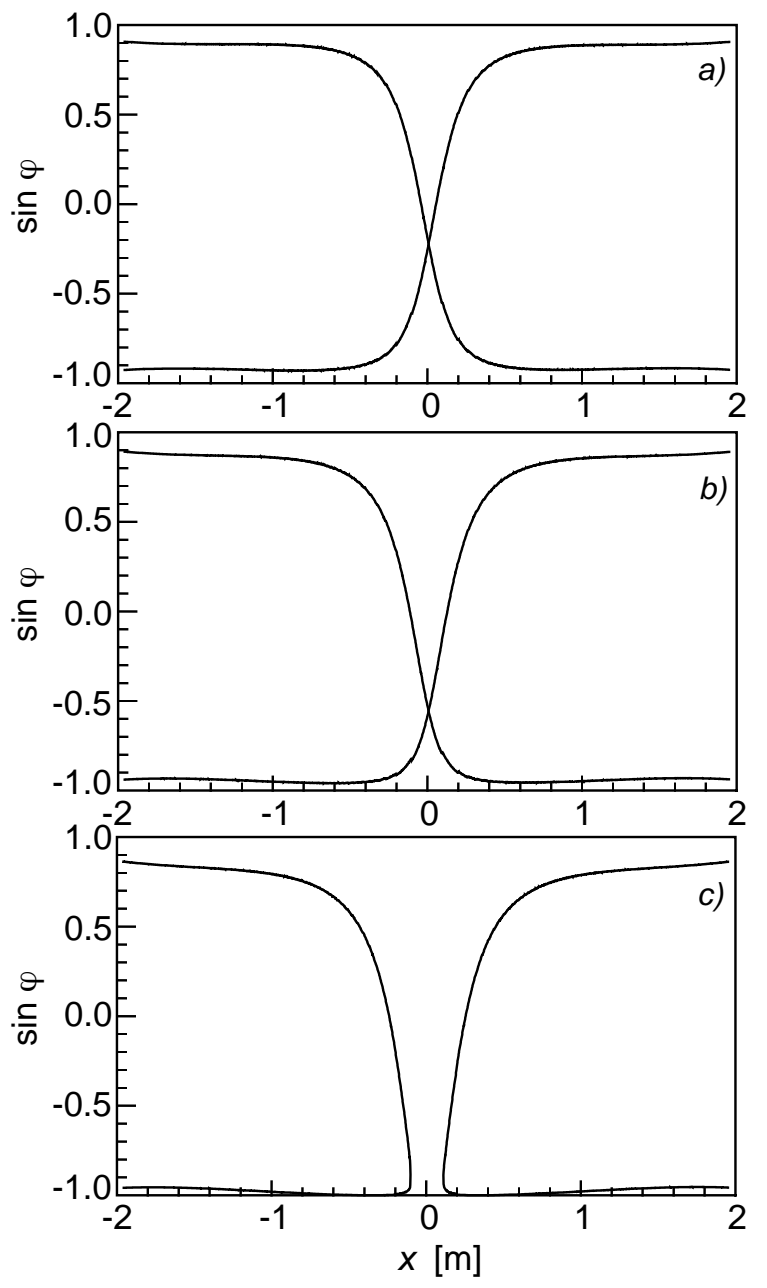

FIG. 7. Value of $\sin \varphi(x)$ along the central ray of the probing beam of Fig. 4 for a constant scattering angle; a) $\left.\left.\theta=1.8^{\circ}, b\right) \theta=4.5^{\circ}, c\right) \theta=9.0^{\circ}$. 

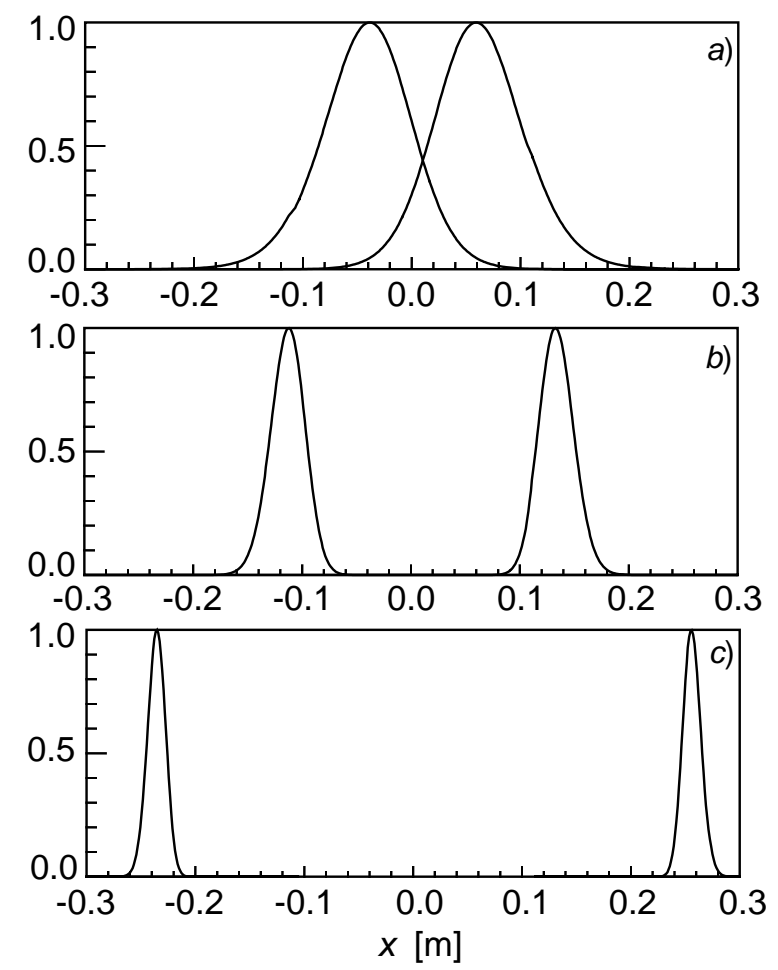

FIG. 8. Instrumental selectivity function for the scattering configuration of Fig. 4 and the scattering angles of Fig. 7; a) $\left.\left.k=2 \mathrm{~cm}^{-1}, b\right) k=5 \mathrm{~cm}^{-1}, c\right) k=10 \mathrm{~cm}^{-1}$. Functions with a maximum at $x<0$ are for $\varphi_{1}=0^{\circ}$, other functions are for $\varphi_{1}=180^{\circ}$. 


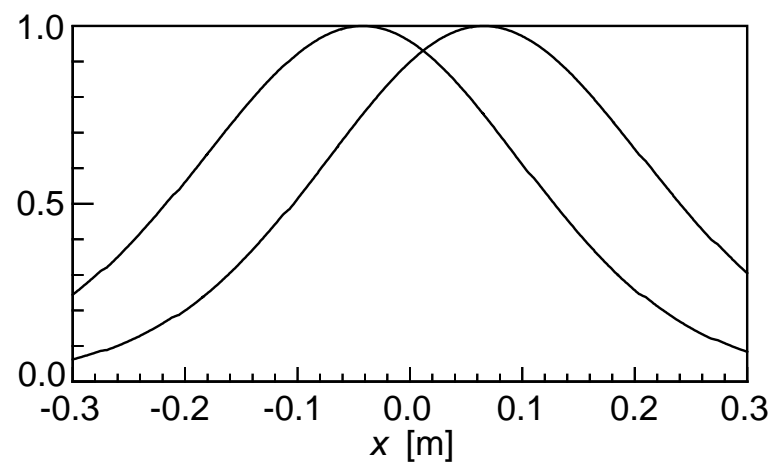

FIG. 9. Same as in case $a$ ) of Fig. 8 but with the opposite value of launching angle $\left(\gamma=4.5^{\circ}\right)$. 

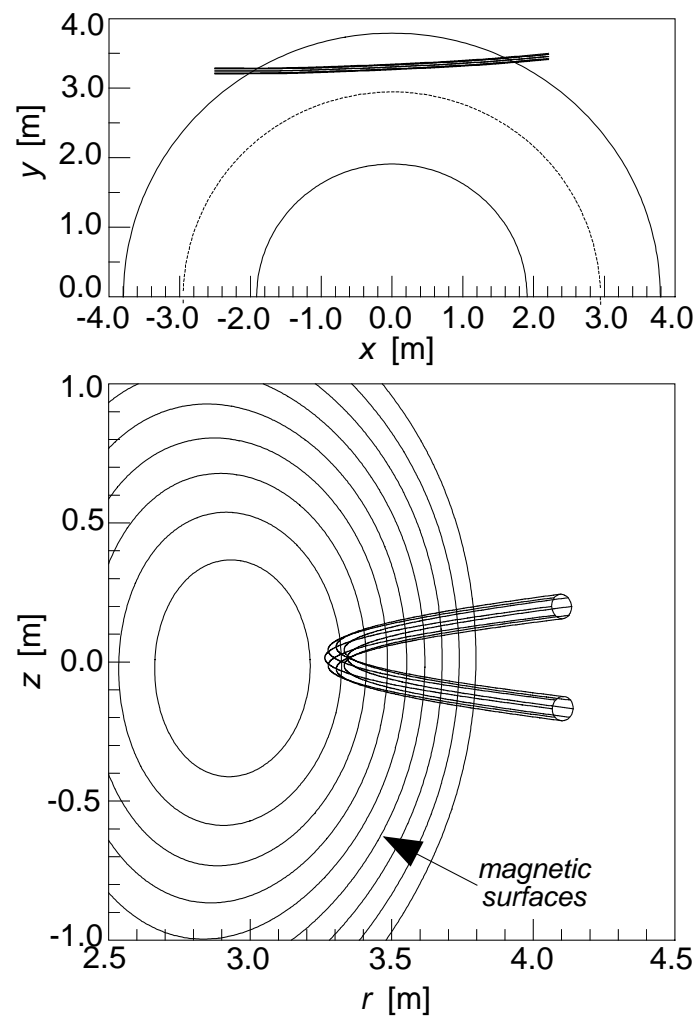

FIG. 10. Same as in Fig. 4 but with wave refraction from a plasma with a peak density of $5 \times 10^{19} \mathrm{~m}^{-3}$ (ordinary mode of propagation). 

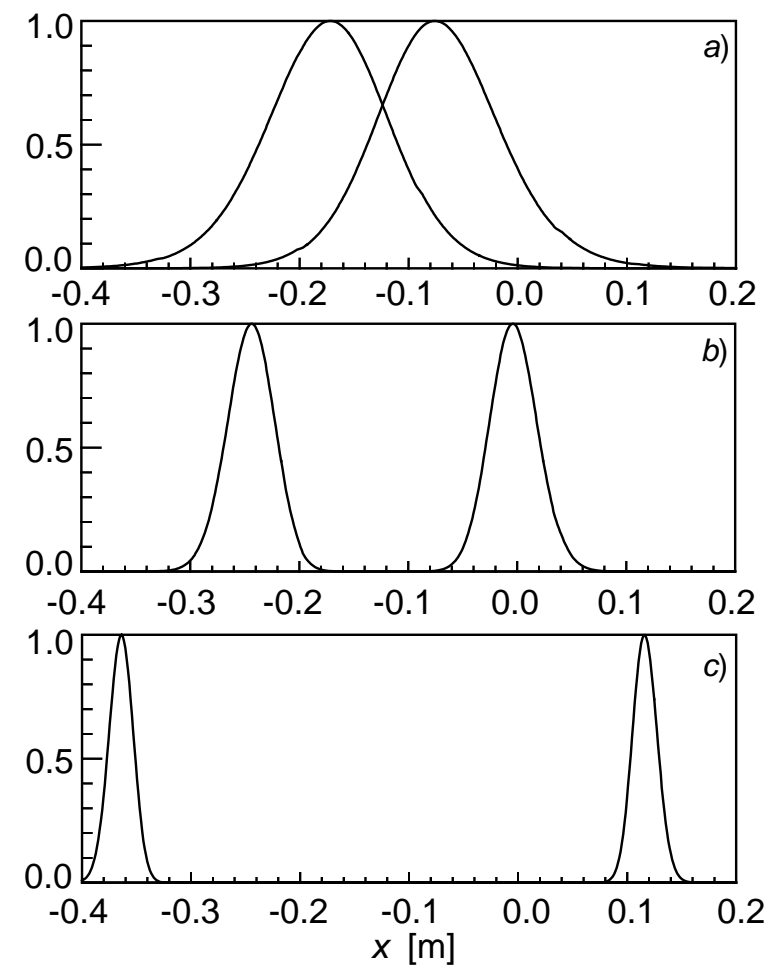

FIG. 11. Same as in Fig. 8 for the scattering geometry of Fig. 10. Beam refraction leads to a shift in the position of the instrumental functions. 

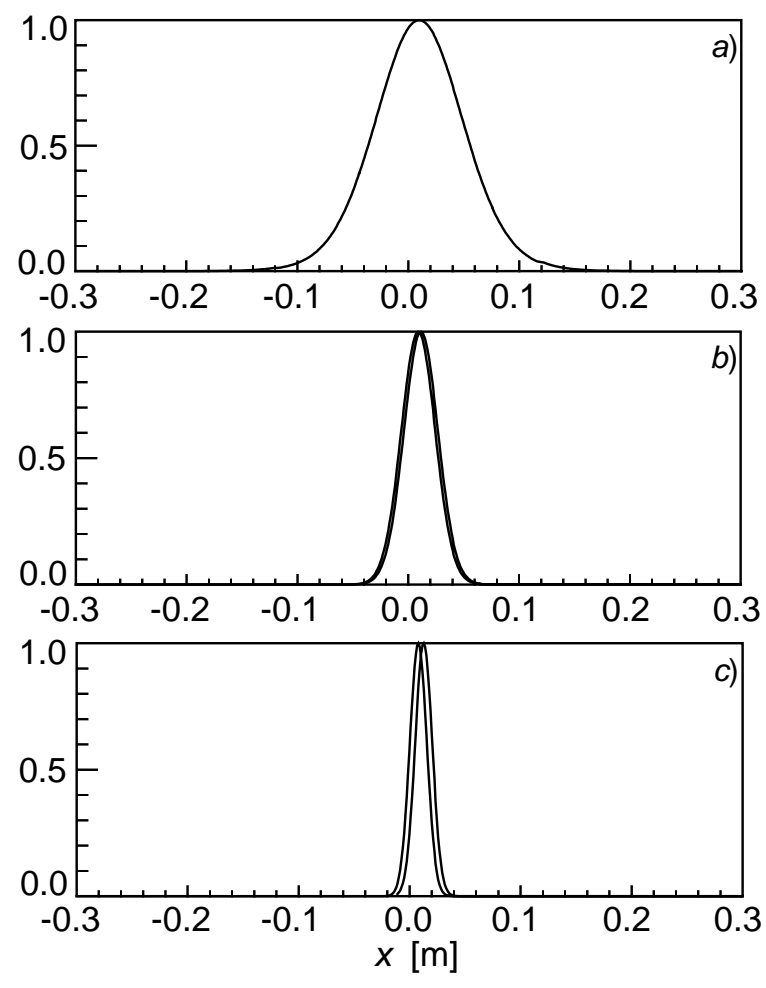

FIG. 12. Same as in Fig. 11 for a probing frequency of $3 \times 10^{13} \mathrm{~Hz}\left(\mathrm{CO}_{2}\right.$ laser $)$. The increased frequency (i.e., small scattering angles) minimizes the shift of the instrumental function away from the tangency point $(x=0)$, thus optimizing the measurement localization. 


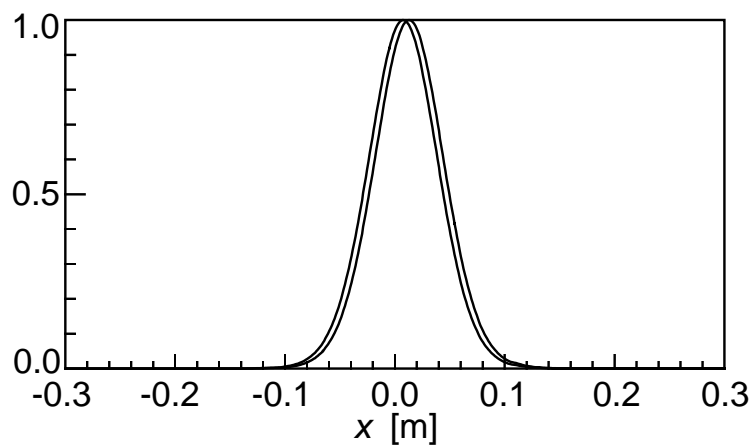

FIG. 13. Same as in case $c$ ) of Fig. 12 for a beam radius of $1 \mathrm{~cm}$. The reduction of the radius simultaneously improves the radial localization $(\sim 2 w)$ and the scattered power. 


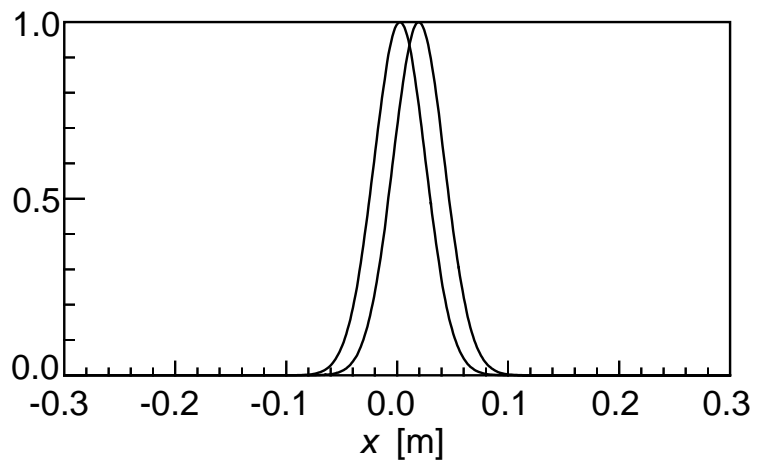

FIG. 14. Instrumental selectivity function for $k=30 \mathrm{~cm}^{-1}, \gamma=0^{\circ}, w=1 \mathrm{~cm}$ and a probing frequency of $3 \times 10^{13} \mathrm{~Hz}\left(\mathrm{CO}_{2}\right.$ laser). An over-reduction in scattering localization due to the large value of $k$ is mitigated by slightly increasing $\alpha_{\text {min }}$, which serves to increase the scattered power. 

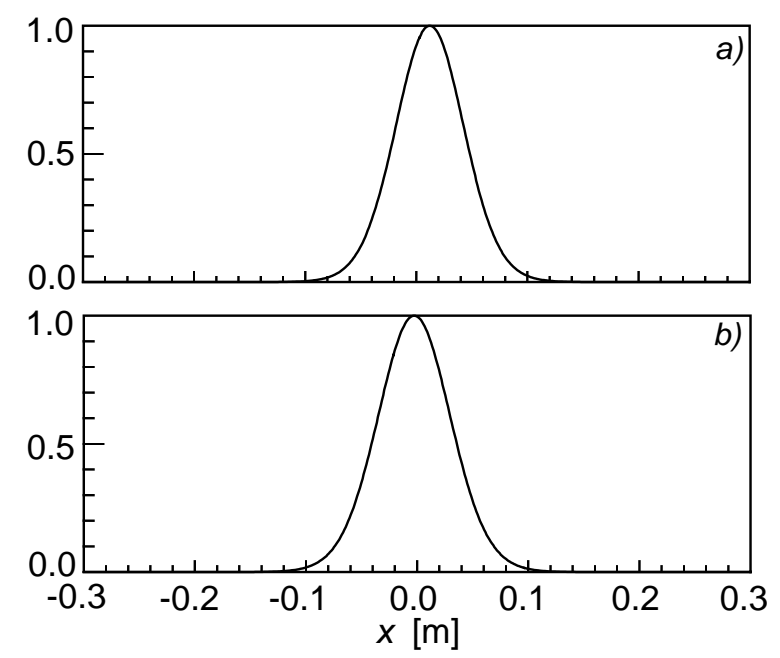

FIG. 15. Instrumental selectivity function for the detection of fluctuations with $k=5$ $\mathrm{cm}^{-1}$ and $\varphi=0^{\circ}$ at $r=3.1 \mathrm{~m}(a)$ and $r=3.6 \mathrm{~m}(b)$. The probing beam has a frequency of $3 \times 10^{13} \mathrm{~Hz}, w=2 \mathrm{~cm}, \gamma=0^{\circ}(a)$ and $\gamma=-12^{\circ}(b)$. 


\section{External Distribution}

Plasma Research Laboratory, Australian National University, Australia

Professor I.R. Jones, Flinders University, Australia

Professor João Canalle, Instituto de Fisica DEQ/IF - UERJ, Brazil

Mr. Gerson O. Ludwig, Instituto Nacional de Pesquisas, Brazil

Dr. P.H. Sakanaka, Instituto Fisica, Brazil

The Librarian, Culham Laboratory, England

Library, R61, Rutherford Appleton Laboratory, England

Mrs. S.A. Hutchinson, JET Library, England

Professor M.N. Bussac, Ecole Polytechnique, France

Librarian, Max-Planck-Institut für Plasmaphysik, Germany

Jolan Moldvai, Reports Library, MTA KFKI-ATKI, Hungary

Dr. P. Kaw, Institute for Plasma Research, India

Ms. P.J. Pathak, Librarian, Insitute for Plasma Research, India

Ms. Clelia De Palo, Associazione EURATOM-ENEA, Italy

Dr. G. Grosso, Instituto di Fisica del Plasma, Italy

Librarian, Naka Fusion Research Establishment, JAERI, Japan

Library, Plasma Physics Laboratory, Kyoto University, Japan

Research Information Center, National Institute for Fusion Science, Japan

Dr. O. Mitarai, Kyushu Tokai University, Japan

Library, Academia Sinica, Institute of Plasma Physics, People's Republic of China

Shih-Tung Tsai, Institute of Physics, Chinese Academy of Sciences, People's Republic of China

Dr. S. Mirnov, TRINITI, Troitsk, Russian Federation, Russia

Dr. V.S. Strelkov, Kurchatov Institute, Russian Federation, Russia

Professor Peter Lukac, Katedra Fyziky Plazmy MFF UK, Mlynska dolina F-2, Komenskeho Univerzita, SK-842 15 Bratislava, Slovakia

Dr. G.S. Lee, Korea Basic Science Institute, South Korea

Mr. Dennis Bruggink, Fusion Library, University of Wisconsin, USA

Institute for Plasma Research, University of Maryland, USA

Librarian, Fusion Energy Division, Oak Ridge National Laboratory, USA

Librarian, Institute of Fusion Studies, University of Texas, USA

Librarian, Magnetic Fusion Program, Lawrence Livermore National Laboratory, USA

Library, General Atomics, USA

Plasma Physics Group, Fusion Energy Research Program, University of California at San Diego, USA

Plasma Physics Library, Columbia University, USA

Alkesh Punjabi, Center for Fusion Research and Training, Hampton University, USA

Dr. W.M. Stacey, Fusion Research Center, Georgia Institute of Technology, USA

Dr. John Willis, U.S. Department of Energy, Office of Fusion Energy Sciences, USA

Mr. Paul H. Wright, Indianapolis, Indiana, USA 
The Princeton Plasma Physics Laboratory is operated by Princeton University under contract with the U.S. Department of Energy.

\author{
Information Services \\ Princeton Plasma Physics Laboratory \\ P.O. Box 451 \\ Princeton, NJ 08543
}

Phone: 609-243-2750

Fax: 609-243-2751

e-mail: pppl_info@pppl.gov

Internet Address: http://www.pppl.gov 